\title{
Risk factors and basic mechanisms of chronic thromboembolic pulmonary hypertension: a current understanding
}

\author{
Irene M. Lang*, Raffaele Pesavento\#, Diana Bonderman* and Jason X-J. Yuan`
}

ABSTRACT: All available evidence today indicates that chronic thromboembolic pulmonary hypertension (CTEPH) is primarily caused by venous thromboembolism, as opposed to primary pulmonary vascular in situ thrombosis. Both the initial magnitude of clot and pulmonary embolism (PE) recurrence may contribute to the development of CTEPH. Only few specific thrombophilic factors, such as phospholipid antibodies, lupus anticoagulant and elevated factor VIII, are statistically associated with CTEPH.

A mechanistic view of CTEPH as a disease caused by obliteration of central pulmonary arteries by pulmonary emboli is too simplistic. Based on available data one may speculate that PE may be followed by a pulmonary vascular remodelling process modified by infection, immune phenomena, inflammation, circulating and vascular-resident progenitor cells, thyroid hormone replacement or malignancy. Both plasmatic factors (hypercoagulation, "sticky" red blood cells, high platelet counts and uncleavable fibrinogens) and a misguided vascular remodelling process contribute to major vessel and small vessel obliteration. Endothelial dysfunction and endothelialmesenchymal transition may be important, but their precise roles remain obscure. There exists no animal model for CTEPH; therefore, experimentation in the future must include human tissues and clinical data in parallel.

KEYWORDS: Chronic thromboembolic pulmonary hypertension, coagulation, review, vascular biology

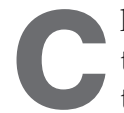
hronic thromboembolic pulmonary hypertension $(\mathrm{CTEPH})$ results from the obstruction of predominantly major (central or proximal large) pulmonary vessels by organised blood clots. CTEPH is a common variation of pulmonary hypertension (PH) [1]. Current data derived from registries suggest that CTEPH occurs at an incidence of 3-30 per million in the general population. Although CTEPH does not show the classical risk profile of venous thromboembolism (VTE), the disease has been demonstrated to emerge as a long-term complication of symptomatic pulmonary embolism (PE) with a cumulative incidence of $0.1-9.1 \%$ within 2 yrs of the event [2-11]. Still, a significant number of cases may originate from asymptomatic VTE [1, 12].

\section{DEFINITION}

CTEPH is defined by the following observations after $\geqslant 3$ months of effective anticoagulation [13]: 1) mean pulmonary arterial pressure $>25 \mathrm{mmHg}$ with a pulmonary capillary wedge pressure $\leqslant 15 \mathrm{mmHg}$; and 2) at least one (segmental) perfusion defect detected by lung scanning, multidetector computed tomographic angiography or pulmonary angiography.

Although CTEPH is considered a venous thromboembolic disorder, classic thromboembolic plasmatic risk factors are lacking. Over the past few years, several important observations and clarifications have been made with regard to a better

\section{THE THROMBOEMBOLIC CONCEPT}

Previous articles in this series. No. 1: Delcroix M, Vonk Nordegraaf A, Fadel E, et al. Vascular and right ventricular remodelling in chronic thromboembolic

\section{AFFILIATIONS}

*Dept of Internal Medicine II, Division of Cardiology, Vienna General Hospital, Medical University of Vienna, Vienna, Austria.

\#Dept of Cardiothoracic and Vascular Sciences, University of Padua, Padua, Italy.

"Dept of Medicine, University of Illinois at Chicago, Chicago, IL, USA.

\section{CORRESPONDENCE}

I.M. Lang

Dept of Internal Medicine ॥ Division of Cardiology Medical University of Vienna Währinger Gürtel 18-20 1090 Vienna Austria E-mail: irene.lang@ meduniwien.ac.at 
understanding of the disease [1]. In previous pathology series, $<50 \%$ of patients had an identifiable coagulation, autoimmune or haematological abnormality [14, 15]. However, the European CTEPH Registry has recently revealed that previous PE is detected in $74.8 \%$ of all CTEPH patients while previous deep venous thrombosis (DVT) is documented in $56.1 \%$ of patients [16]. Based on these data, it appears adequate to view CTEPH as a sequela of classical thromboembolism that is modified by misguided thrombus resolution leading to occlusive vascular remodelling (fig. 1) in proximal and distal vessels. Moreover, some, but not all, risk factors of recurrent VTE are present, such as elevated factor VIII (FVIII) [17] and lupus anticoagulant (LAC)/ antiphospholipid antibodies (APAs) [18]. In contrast to pulmonary arterial hypertension (PAH) involving vessels $<300 \mu \mathrm{m}$ in diameter, CTEPH typically affects major vessels [13] and is therefore amenable to surgical removal of the obstructions [19], i.e. pulmonary endarterectomy (PEA). Figure 2 shows a typical specimen surgically removed from a CTEPH patient, representing a cast of the pulmonary vascular bed, consisting of endothelial cells, smooth muscle cells, fibroblasts and fresh thrombus by histological examination [1]. While the concept of major-vessel disease (i.e. CTEPH) and small-vessel disease (i.e. $\mathrm{PAH}$ ) sounds intriguing, there are cases of CTEPH overlapping with $\mathrm{PAH}$, in that they are characterised by distal thromboembolic lesions and a typical pulmonary arteriopathy. Depending on the assessment of the surgical team, those cases are classified as inoperable CTEPH.

\section{COAGULATION AND FIBRINOLYSIS}

A low incidence of CTEPH after PE, a lack of symptomatic PE in $\geqslant 25 \%$ of patients with CTEPH and the absence of classical thromboembolic risk factors challenges the link between PE and CTEPH [1, 20, 21]. For example, a deficiency of antithrombin, protein C and protein S, the G20210A mutation of prothrombin and hyperhomocysteinaemia are not associated with CTEPH. The factor V R506Q mutation (factor V Leiden) is not more common in CTEPH [18]. However, in a recent retrospective study, factor $\mathrm{V}$ Leiden was more frequent in CTEPH than in other $\mathrm{PH}(29 \%$ versus $7.8 \% ; \mathrm{p}=0.001)$, and the prothrombin mutation was more frequent in $\mathrm{CTEPH}$, albeit not significantly

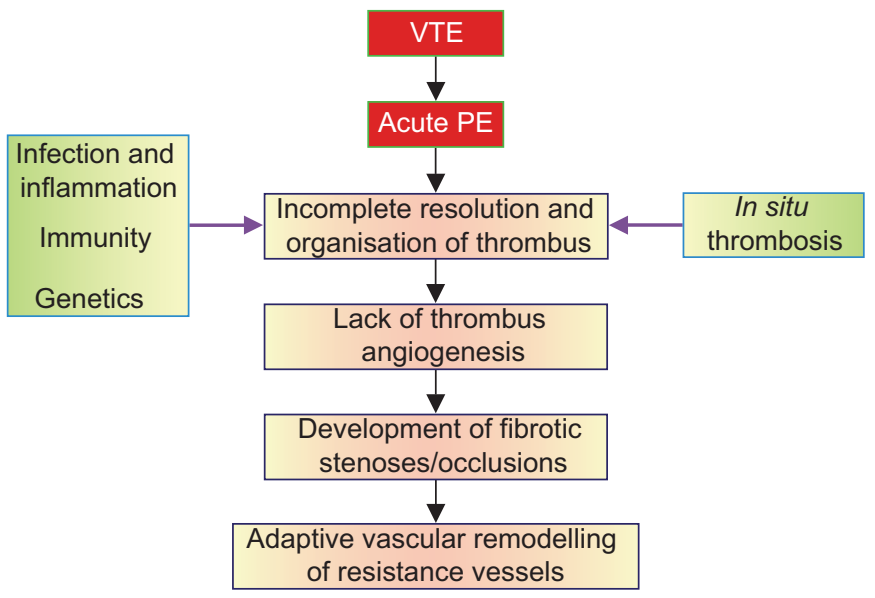

FIGURE 1. Scheme of the pathophysiological concept of chronic thromboembolic pulmonary hypertention. VTE: venous thromboembolism; PE: pulmonary embolism.
[22]. Factor $\mathrm{V}$ mutation prevalence is dependent on the populations that are studied. The association between combined defects and CTEPH is anecdotal [23]. LACs/APAs and the coagulation factor FVIII have been found associated with VTE and CTEPH $[13,18,21,22,24,25]$.

APAs have been detected in patients with various forms of $\mathrm{PH}$, but usually in low titres. High APA titres were found in CTEPH patients, and high titres were associated with LAC [18]. In patients with CTEPH, the detection of LAC suggests a prothrombotic state; this may not be true in patients with nonthromboembolic $\mathrm{PH}$ in whom other immunological and inflammatory mechanisms may be involved [26, 27]. The finding of multiple positive tests in CTEPH patients is of interest because of a recent publication illustrating a higher risk of VTE recurrence in triple-positive tests [28, 29].

Plasma concentrations of FVIII and von Willebrand factor antigen (VWF:Ag) were measured in 122 consecutive patients with CTEPH, in 88 patients with nonthromboembolic PAH, and in 82 age- and sex-matched healthy controls [17]. Elevated VWF:Ag and FVIII $\left(>230 \mathrm{IU} \cdot \mathrm{dL}^{-1}\right)$ were more common in patients with CTEPH than in both $\mathrm{PAH}$ patients and controls ( $41 \%$ versus $5 \%$ and $22 \%$, respectively). After successful PEA, no decrease in FVIII plasma concentrations occurred; in contrast, plasma FVIII decreases after alleviation of $\mathrm{PH}$ by prostanoid treatment [30]. These data suggest that elevated values of FVIII in patients with CTEPH might be causal to the disease process.

Acute episodes of VTE may be associated with the risk of developing CTEPH. In a prospective cohort study, 305 patients with symptomatic VTE were recruited, 223 with a first episode of PE, 58 with previous DVT and 24 with previous PE [8]. The incidence of CTEPH in the first group was 3.8\% within the first 2 yrs of follow-up. In the other two groups, cumulative incidences of CTEPH were $5.2 \%$ and $33.3 \%$, respectively. In the multivariate logistic regression model, younger age (OR 1.79, 95\% CI 1.23-2.56), previous PE (OR 19, 95\% CI 4.5-79.8), an idiopathic clinical presentation of PE (OR 5.70, 95\% CI 1.41-22 .97) and larger perfusion defects at the time of PE diagnosis (OR 2.22, 95\% CI 1.49-3.31) were associated with an increased risk of CTEPH [8]. In a recent smaller prospective cohort study on 110 patients

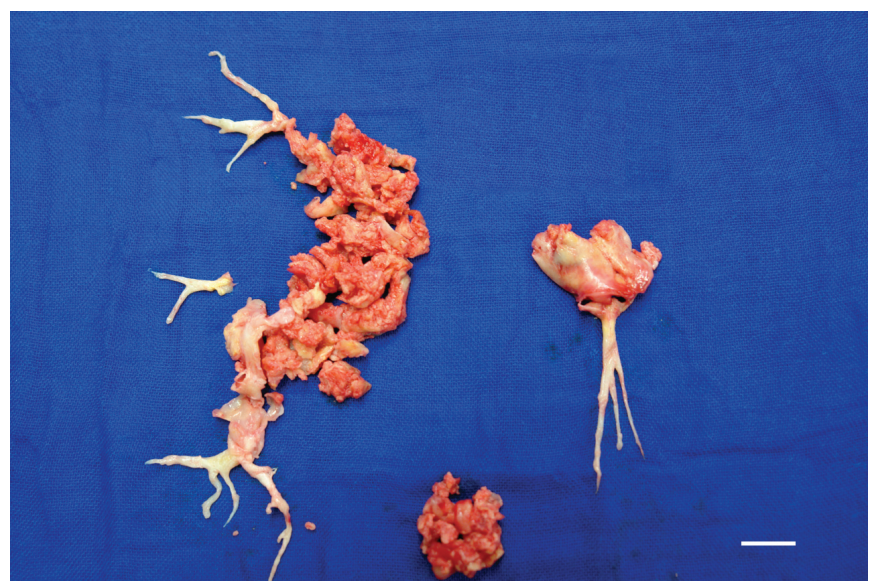

FIGURE 2. Major vessel obstructions ("acute and chronic clot") removed by pulmonary endarterectomy from the right and left pulmonary artery. Scale bar $=1 \mathrm{~cm}$ 
with acute PE, CTEPH incidence was 9.1\% within 2 yrs; six (5.4\%) cases were diagnosed during variable clinical follow-up and the remaining cases were diagnosed on the basis of echocardiographic and clinical findings at 2 yrs from the index event [11]. In the multivariate regression model older age (relative risk (RR) 1.2 per year, 95\% CI 1.0-1.3) and a combination of previous and recurrent PE were found to be associated with CTEPH (RR 5.7, 95\% CI 1.5-22.0). In a large retrospective cohort study, 687 consecutive patients with $\mathrm{PH}$ were enrolled in four large European databases [25]. 433 of these patients were diagnosed with CTEPH and 254 with nonthromboembolic PH. A logistic regression analysis was performed in 585 patients showing that both previous and recurrent VTE were significantly associated with an increased risk of CTEPH (OR 4.52, 95\% CI 2.35-9.12 and OR $14.49,95 \%$ CI 5.40-43.08, respectively). Even chronic venous ulcers, which can be viewed as the late sequelae of DVT, were more common in CTEPH patients (OR 2.84, 95\% CI 0.99-9.00).

In a prospective cohort study on 254 subjects observed for a median 3.8 yrs after a first episode of acute PE, 0.8\% developed $\mathrm{CTEPH} \mathrm{[3].} \mathrm{Interestingly,} \mathrm{this} \mathrm{incidence} \mathrm{increased} \mathrm{to} 1.5 \%$ if only patients with an idiopathic clinical presentation of PE were considered, which is similar to that observed by PENGO et al. [8], although no VTE recurrences were observed in patients diagnosed with CTEPH. However, the exclusion of both cancer patients and those with major thrombophilic disorders, the relatively low severity of acute PE episodes and the very careful management of anticoagulant therapy may have influenced the true incidence of CTEPH. In another prospective cohort study of 834 subjects with suspected PE, 320 patients with an objectively confirmed diagnosis were followed-up for a median 2.1 yrs [6]. This study was unique in that it performed serial lung scans in all patients. The incidence of CTEPH was 1.3\%; the median extent of pulmonary vascular obstruction was almost $60 \%$ in patients diagnosed with CTEPH. There was no evidence of recurrent PE in serial lung scans. However, an association between recurrent PE and CTEPH could have been underestimated: 1-yr mortality was high, and three out of four CTEPH patients had had documented episodes of DVT without any objective imaging tests for PE before entering the study. PoLI et al. [31] prospectively observed a cohort of 239 patients with a first episode of PE and found a $0.4 \%$ CTEPH incidence after a median follow-up of 3 yrs. VTE recurrences and high levels of D-dimer after withdrawal of anticoagulant therapy were not associated with an increased risk of CTEPH. Notably, none of the patients with VTE recurrences had high systolic pulmonary arterial pressure. However, the lack of data about the severity of the index event and the exclusion of patients with cancer introduced a selection bias. In up to $14 \%$ of subjects a treatment strategy based on D-dimer testing was adopted in order to reduce VTE recurrences, and this may have affected CTEPH incidence. KLOK et al. [5] investigated the occurrence of CTEPH in 866 unselected consecutive patients diagnosed with PE. Provided that a diagnosis of CTEPH was not made, the 588 survivors underwent an echocardiographic study after a median time of 3.7 yrs after the index event; in case of suspected CTEPH a diagnostic procedure was performed for confirmation purposes. The cumulative incidence of CTEPH was $0.57 \%$ in the overall population and $1.5 \%$ in patients with unprovoked PE. Recurrent PE was not associated with a higher risk of developing CTEPH. However, a lack of data related to the acute index event, variable duration of follow-up and the exclusion of $\sim 30 \%$ of eligible subjects significantly limited the power of detecting CTEPH.

RIEDEL et al. [20] assessed haemodynamics at $\geqslant 2$ months after acute PE in 76 patients. A haemodynamic investigation was subsequently performed in subjects surviving 1-15 yrs after the first examination [32]. The detection of a mean pulmonary artery pressure $>30 \mathrm{mmHg}$ at baseline was associated with a further increase during follow-up (from $47.1 \pm 14.6$ to $55.3 \pm 10.1 \mathrm{mmHg}$, $\mathrm{p}<0.05$ ), while normal mean pulmonary artery pressures showed no late increase.

In a small prospective cohort study RIBEIRO et al. [7] performed serial echocardiographic examinations within $1 \mathrm{yr}$ of the acute event in 78 patients who were subsequently followed over 5 yrs. The detection of systolic pulmonary artery pressures $>50 \mathrm{mmHg}$ at the time of the initial diagnosis of PE was associated with a three-fold increase in the risk of $\mathrm{PH}$ at 1 yr [7]. Notably, three out of four patients with persistent $\mathrm{PH}$ underwent PEA. The detection of right ventricular dysfunction during the acute phase of PE in a cohort of 301 patients with a first acute episode of PE was associated with an almost four-fold risk of VTE recurrences after a 3-yr follow-up (hazard ratio 3.79, 95\% CI 1.8-7.8) [33]. However, the question remains whether early detection of significantly elevated pulmonary arterial pressures is related to true acute $\mathrm{PE}$ with right ventricular strain, or to an early manifestation of CTEPH.

Taken together, currently available evidence confirms that at least some prothrombotic risk factors of acute and recurrent VTE are prevalent in CTEPH patients, while fibrinolytic factors have been shown to be bystanders rather than mediators of the disease [34]. In general, the clinical presentation of the acute PE episode, and possibly VTE recurrences, increase the risk of developing СТЕРН. Recent studies have demonstrated that persistent residual thrombotic material in the deep venous system is associated with a higher risk of VTE recurrences and identify a subgroup of patients in whom prolonging anticoagulant therapy is beneficial [35].

Current evidence suffers from several limitations: patient cohorts are heterogeneous and their mortality rates variable; studies are often retrospective and not adequately controlled; the severity of the initial embolic episode is often unknown; and it is very hard to exclude pre-existing PH. However, the recent description of the European patient cohort indicating that previous PE was confirmed in three-quarters of patients and previous VTE was observed in half of them provides firm evidence for the thromboembolic nature of the disease, despite continued controversy over this issue [36].

\section{OTHER RISK FACTORS}

Nonplasmatic risk factors such as splenectomy, ventriculoatrial (VA) shunt for hydrocephalus therapy, inflammatory bowel disease and chronic osteomyelitis are associated with a higher incidence and a worse prognosis of CTEPH [21, 25, 37]. In a recent retrospective database involving three European PEA centres, data from $687 \mathrm{CTEPH}$ patients were compared with nonthromboembolic precapillary PAH cohorts at the participating institutions. VA shunts and infected pacemakers (OR 76.40, 95\% CI 7.67-10351; p<0.001), splenectomy (OR 17.87, 95\% CI $1.56-2438 ; \mathrm{p}=0.017$ ), and non-O blood groups (OR 2.09, 95\% CI 1.12-3.94; $\mathrm{p}=0.019)$ were more often associated with CTEPH. 
Thyroid replacement therapy (OR 6.10, 95\% CI 2.73-15.05; $\mathrm{p}<0.001$ ) and a history of malignancy (OR 3.76, 95\% CI 1.4710.43; $\mathrm{p}=0.005)$ emerged as novel CTEPH risk factors. Recent data from the French $\mathrm{PH}$ programme reporting on 17 cases of Port-A-Cath ${ }_{\circledast}$ (Smiths Medical, Grasbrunn, Germany) central venous access systems entailing CTEPH [38] with 30\% culturepositive for Staphylococcus epidermidis lends support to the concept of infected intravenous lines as a pathological trigger for CTEPH.

\section{PATHOPHYSIOLOGICAL CONCEPTS AND ANIMAL MODELS}

The concept of "inflammatory thrombosis"

In the majority of subjects, venous thrombi are removed by complex processes of degradation and organisation, including adaptive (mostly positive) remodelling of the vessel wall and recanalisation. This process is very similar to the formation of granulation tissue during wound healing. Leukocyte recruitment and angiogenesis play important roles during thrombus resolution. Initially neutrophils and subsequently monocytes are recruited into organising thrombi. Thrombus resolution is severely impaired when monocyte recruitment is reduced. Monocytes are capable of expressing and secreting a host of chemo-attractants (e.g. monocyte chemotactic protein (MCP)-1), growth factors (e.g. vascular endothelial growth factor (VEGF), basic fibroblast growth factor (bFGF), transforming growth factor (TGF)- $\alpha$ and - $\beta 1$ ) and proteases (e.g. urokinase-type plasminogen activator and matrix metalloproteinases). Inflammatory markers, e.g. C-reactive protein (CRP) [26], tumour necrosis factor- $\alpha$ [39] and MCP-1 [40] have been found to be elevated in plasma and thrombus tissues of patients with CTEPH, in correlation with haemodynamics.

\section{The concept of "deficient angiogenesis"}

In CTEPH, angiogenesis depends on vasa vasorum that derive from the systemic bronchial arteries. After pulmonary arterial occlusion, these vessels normally spread into the pulmonary arteries and pre-existing collaterals are opened. In the course of healing or thrombus organisation, positive regulators of angiogenesis predominate and the endothelium becomes activated, penetrating the occlusive thrombus [41]. Previous animal studies have demonstrated that recanalisation of the vein wall may occur within $24 \mathrm{~h}$ of thrombus formation. During the early phase of resolution, thrombi begin to contract and retract from the vein wall, which results in the formation of cell-lined pockets between the body of the thrombus and the intima of the vein wall. The appearance of vascular channels is associated with the expression of VEGF and bFGF, which are thought to drive neovascularisation within the thrombus [42]. Collagen-secreting cells were detected in PEA material [43], participating in the formation of a microenvironment that leads to dysfunctional endothelial cells that do not support the angiogenetic process [44]. Previous animal studies demonstrated that VEGF administration enhanced thrombus resolution in animal models $[45,46]$. Nevertheless, the observation was rejected by certain experimental setups [47]. Recent experimental data suggest that stimulating hypoxiainducible factor- $1 \alpha$ levels in the vein wall leads to an increased angiogenic drive and promotes vein recanalisation and thrombus resolution [48]. These observations, and the deficiency of angiogenetic gene expression in CTEPH obstructions detected by differential display analysis and RT-PCR, suggest that the failure to resolve pulmonary vascular obstructions may be interconnected with a deficiency in angiogenesis.

\section{The concept of "abnormal fibrinogen"}

Abnormal variants of fibrinogen [49, 50] and abnormal fragmentation of fibrinogen by plasminogen activators [51] are alternative pathophysiological targets. In a recent study, a significant difference was demonstrated in fibrinogen $(\mathrm{Fg})-\mathrm{A} \alpha$ Thr312Ala genotype and allele frequencies between CTEPH subjects and controls [52]. In addition, the Fg-A $\alpha$ Thr312Ala allele has been associated with an increased risk of VTE [53].

\section{Animal models}

Animal models have been designed to imitate the thrombosis and thrombus resolution pathways in the pulmonary vasculature, albeit with limited success [54,55]. A porcine model

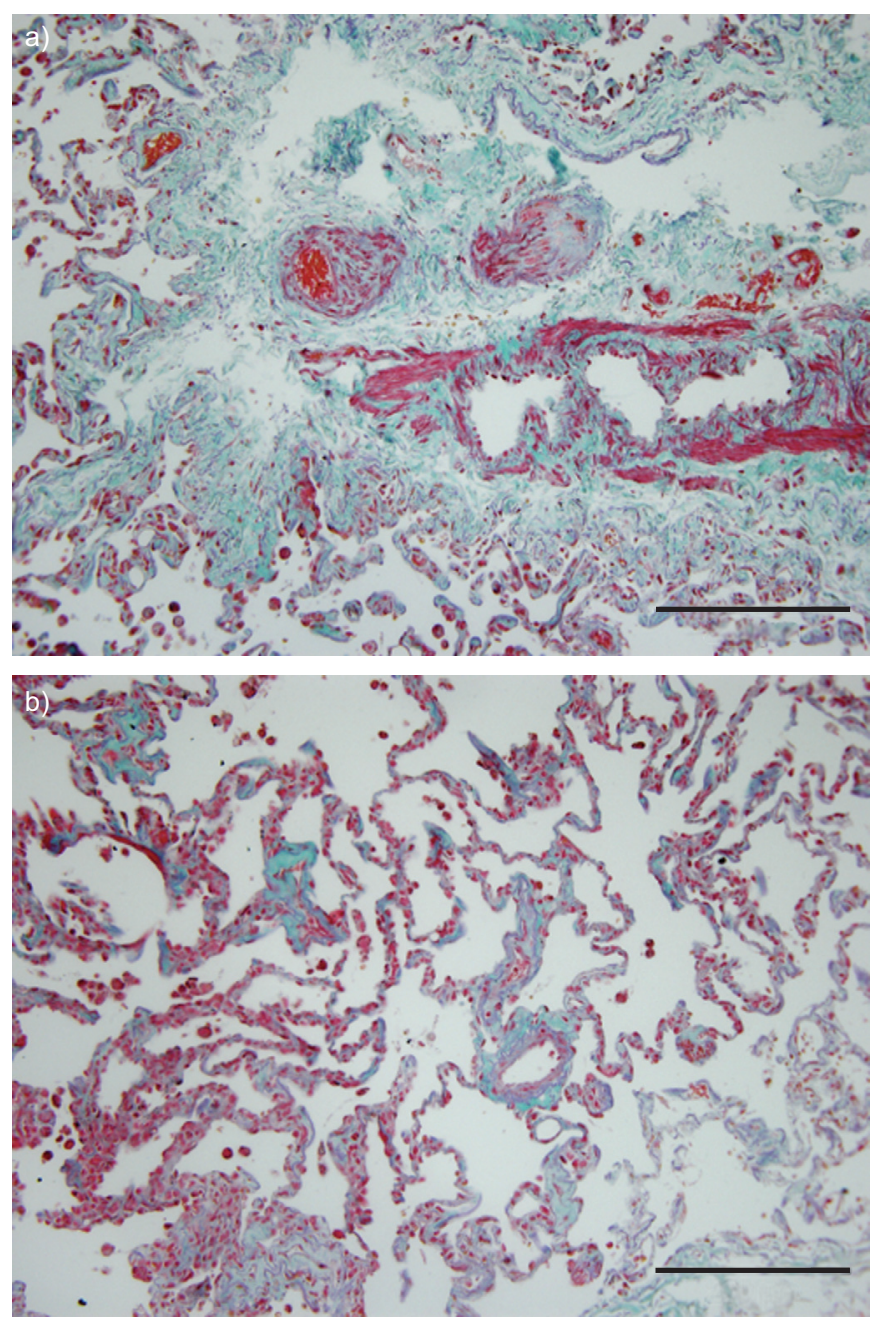

FIGURE 3. "Secondary vascular disease" of chronic thromboembolic pulmonary hypertension observed in lung biopsies obtained during pulmonary endardectomy in the course of a clinical study. a) Trichrome stain of small pulmonary arteries $<200 \mu \mathrm{m}$ in diameter with intimal fibrosis and smooth muscle hypertrophy; b) lung specimen from a lobe dependent upon a completely occluded main pulmonary artery. Note a paucity of vessels. Existing vessels do not display smooth muscle cell hypertrophy and intimal lesions. Both lung specimens are from the same patient (different from that shown in fig. 2). Scale bars $=200 \mu \mathrm{m}$. 
originating from ligation of the left pulmonary artery through a midline sternotomy combined with repeated embolisation of histoacryl into the right lower lobe over 5 weeks may be promising [56]. Several models utilising percutaneous embolisations of the pulmonary vessels in combination with splenectomy are currently under investigation. Alternatively, a murine vena cava ligation model is allowing mechanisms of thrombus resolution to be pinpointed [48, 57].

\section{VASCULAR PATHOBIOLOGY}

According to current knowledge, CTEPH emerges as a "dual" pulmonary vascular disorder with thrombosis inducing major vessel vascular remodelling, combined with a small pulmonary vessel disease (pulmonary arteriopathy) [1, 58]. Vascular lesions comprise distal extension of muscularisation, concentric laminar intimal fibroelastosis and eccentric intimal fibrosis, medial hypertrophy and occasional plexiform lesions. The latter appear to predominate in areas with patent feeding vessels (fig. 3a), while in areas distal to stenotic or occluded vessels a paucity of vessels is observed (fig. 3b). Pulmonary arteriopathy in CTEPH patients is currently interpreted as the sequela of combined endothelial dysfunction with abnormal accumulation of asymmetric dimethylarginine [59], abnormal proliferation of smooth muscle and endothelial cells, fibroblast and smooth muscle cell migration, inhibited apoptosis in vascular smooth muscle cells and in situ thrombosis [60,61]. Key players in this disturbance are prostacyclin and prostacyclin synthase, which have been found deficient in $\mathrm{PAH}$, and physiologically counteract the effects of RhoA/Rho-kinases, small intracellular guanosine triphosphatases having important signalling functions on tissue factor expression, nuclear factor- $\kappa \mathrm{B}$ induction and cyclin $\mathrm{D}$ transcription. It is not known whether the degree of smallvessel disease may be related to the length of time a patient has PH before diagnosis of CTEPH [62]. Recent data suggest that the incidence of small-vessel disease may be greater in patients with associated medical conditions [37], e.g. VA shunts for the treatment of hydrocephalus, splenectomy, inflammatory bowel disease, low-grade malignancy and thyroid replacement therapy. In addition, pulmonary vascular reactivity, which is thought to reflect the functional responsiveness of resistance vessels, parallels prognosis [63]. In greater detail, a decrease in mean pulmonary artery pressure of $\geqslant 10 \%$ after administration of inhaled nitric oxide at diagnostic right heart catheterisation is a predictor of better prognosis. Recently, the observation of infection with staphylococcal species has been demonstrated both in vivo $[38,57]$ and in a detailed analysis involving a mouse model of stagnant-flow venous thrombosis [57]. Staphylococcus aureus or Staphylococcus epidermidis is responsible for up to half of device infections, and bacterial pathogens promote fibrotic wound healing [64]. In the mouse model, staphylococcal infection delayed thrombus resolution in parallel with upregulation of TGF- $\beta$ and connective tissue growth factor, laying grounds for the assumption that thrombus infection may be associated with thrombus nonresolution. Based on these data, one may speculate that timely antibiotic treatment of major VTE may prevent the development of CTEPH.

Pulmonary vascular wall thickening in CTEPH patients may be due to proliferation of adventitial fibroblasts, circulating progenitor/precursor cells and/or myofibroblasts, creating fibrous plaques with angioneogenesis and cholesterol-containing atherosclerotic plaques [65]. In a large series of thromboembolic obstructions analysed at the University of California at San Diego (San Diego, CA, USA), remodelling of thrombi at various stages, granulomas associated with sarcoidosis, and a rare case showing features of inflammatory myofibroblastic tumour, primary or metastatic malignancies, and isolated pulmonary arteritis were observed [58]. In $15 \%$ of cases significant inflammation was noted [14]. The role of inflammation in CTEPH pathogenesis has been emphasised by studies illustrating the expression of the CRP receptor LOX-1 on cells isolated from the major pulmonary vessels in CTEPH [27]. The cells isolated from endarterectomised tissue from CTEPH patients displayed diverse morphological phenotypes. Immunofluorescent staining using the smooth muscle cell (SMC) marker smooth muscle $\alpha$-actin was detected in most of the cells; however, some cells showed defined actin filament structures, some a more general cytoplasmic staining and others very weak staining. Cells stained very strongly with the fibroblast marker vimentin in addition to markers typical of more primitive mesenchymal precursor/progenitor cells, some of them with hyperproliferative, anchorageindependent, invasive and serum-independent features [66]. Furthermore, RT-PCR confirmed the presence of mRNA transcripts indicating primitive cell types. In vitro, it was demonstrated that myofibroblast-like cells harvested from PEA specimens induced endothelial-to-mesenchymal transition that was effectively inhibited by rapamycin [44]. Similar proliferative features were observed in cells isolated and cultured from the proximal pulmonary arteries during PEA [67]. The data suggest that enhanced or deregulated differentiation of progenitor cells into SMC types may occur in occluded vessels, in addition to transmigration or transdifferentiation of adventitial fibroblasts. These mechanisms characterise both vascular remodelling processes observed in CTEPH $[43,68]$.

\section{CONCLUSION AND OUTLOOK}

CTEPH is a unique subset of $\mathrm{PH}$ because of the asset of curability. It may therefore also serve as an elegant model for studies of right ventricular function and pulmonary vascular tissues. In Europe, the Association for Research in CTEPH has established a unique database and platform to drive research and education in this important subsection of pulmonary vascular medicine.

\section{STATEMENT OF INTEREST}

A statement of interest for I.M. Lang can be found at www.erj. ersjournals.com/site/misc/statements.xhtml

\section{REFERENCES}

1 Lang IM. Chronic thromboembolic pulmonary hypertension - not so rare after all. N Engl J Med 2004; 350: 2236-2238.

2 Fedullo PF, Auger WR, Kerr KM, et al. Chronic thromboembolic pulmonary hypertension. N Engl J Med 2001; 345: 1465-1472.

3 Becattini C, Agnelli G, Pesavento R, et al. Incidence of chronic thromboembolic pulmonary hypertension after a first episode of pulmonary embolism. Chest 2006; 130: 172-175.

4 Benotti JR, Ockene IS, Alpert JS, et al. The clinical profile of unresolved pulmonary embolism. Chest 1983; 84: 669-678.

5 Klok FA, van Kralingen KW, van Dijk AP, et al. Prospective cardiopulmonary screening program to detect chronic thromboembolic pulmonary hypertension in patients after acute pulmonary embolism. Haematologica 2010; 95: 970-975. 
6 Miniati M, Monti S, Bottai M, et al. Survival and restoration of pulmonary perfusion in a long-term follow-up of patients after acute pulmonary embolism. Medicine (Baltimore) 2006; 85: 253-262.

7 Ribeiro A, Lindmarker P, Johnsson $\mathrm{H}$, et al. Pulmonary embolism: one-year follow-up with echocardiography doppler and five-year survival analysis. Circulation 1999; 99: 1325-1330.

8 Pengo V, Lensing $\mathrm{AW}$, Prins $\mathrm{MH}$, et al. Incidence of chronic thromboembolic pulmonary hypertension after pulmonary embolism. N Engl J Med 2004; 350: 2257-2264.

9 Dentali F, Donadini M, Gianni M, et al. Incidence of chronic pulmonary hypertension in patients with previous pulmonary embolism. Thromb Res 2009; 124: 256-258.

10 Surie S, Gibson NS, Gerdes VE, et al. Active search for chronic thromboembolic pulmonary hypertension does not appear indicated after acute pulmonary embolism. Thromb Res 2010; 125 e202-e205.

11 Marti D, Gomez V, Escobar C, et al. Incidencia de hipertensión pulmonar tromboembólica crónica sintomática y asintomática [Incidence of symptomatic and asymptomatic chronic thromboembolic pulmonary hypertension]. Arch Broncopneumol 2010; 46: 628-633.

12 Dartevelle P, Fadel E, Mussot S, et al. Chronic thromboembolic pulmonary hypertension. Eur Respir J 2004; 23: 637-648.

13 Moser KM, Auger WR, Fedullo PF. Chronic major-vessel thromboembolic pulmonary hypertension. Circulation 1990; 81: 1735-1743.

14 Bernard J, Yi ES. Pulmonary thromboendarterectomy: a clinicopathologic study of 200 consecutive pulmonary thromboendarterectomy cases in one institution. Hum Pathol 2007; 38: 871-877.

15 Blauwet LA, Edwards WD, Tazelaar HD, et al. Surgical pathology of pulmonary thromboendarterectomy: a study of 54 cases from 1990 to 2001. Hum Pathol 2003; 34: 1290-1298.

16 Pepke-Zaba J, Delcroix M, Lang I, et al. Chronic thromboembolic pulmonary hypertension (CTEPH): results from an international prospective registry. Circulation 2011; 124: 1973-1981.

17 Bonderman D, Turecek PL, Jakowitsch J, et al. High prevalence of elevated clotting factor VIII in chronic thromboembolic pulmonary hypertension. Thromb Haemost 2003; 90: 372-376.

18 Wolf M, Boyer-Neumann C, Parent F, et al. Thrombotic risk factors in pulmonary hypertension. Eur Respir J 2000; 15: 395-399.

19 Jamieson SW, Kapelanski DP, Sakakibara N, et al. Pulmonary endarterectomy: experience and lessons learned in 1,500 cases. Ann Thorac Surg 2003; 76: 1457-1462.

20 Riedel M, Stanek V, Widimsky J, et al. Longterm follow-up of patients with pulmonary thromboembolism. Late prognosis and evolution of hemodynamic and respiratory data. Chest 1982; 81: 151-158.

21 Bonderman D, Jakowitsch J, Adlbrecht C, et al. Medical conditions increasing the risk of chronic thromboembolic pulmonary hypertension. Thromb Haemost 2005; 93: 512-516.

22 Wong CL, Szydlo R, Gibbs S, et al. Hereditary and acquired thrombotic risk factors for chronic thromboembolic pulmonary hypertension. Blood Coagul Fibrinolysis 2010; 21: 201-206.

23 Laczika K, Lang IM, Quehenberger $\mathrm{P}$, et al. Unilateral chronic thromboembolic pulmonary disease associated with combined inherited thrombophilia. Chest 2002; 121: 286-289.

24 Auger WR, Permpikul P, Moser KM. Lupus anticoagulant, heparin use, and thrombocytopenia in patients with chronic thromboembolic pulmonary hypertension: a preliminary report. Am J Med 1995; 99: 392-396.

25 Bonderman D, Wilkens H, Wakounig S, et al. Risk factors for chronic thromboembolic pulmonary hypertension. Eur Respir J 2009; 33: 325-331.

26 Quarck R, Nawrot T, Meyns B, et al. C-reactive protein: a new predictor of adverse outcome in pulmonary arterial hypertension. J Am Coll Cardiol 2009; 53: 1211-1218.
27 Wynants M, Quarck R, Ronisz A, et al. Effects of C-reactive protein on human pulmonary vascular cells in chronic thromboembolic pulmonary hypertension. Eur Respir J 2012; 40: 886-894.

28 Pengo V, Ruffatti A, Legnani C, et al. Clinical course of high-risk patients diagnosed with antiphospholipid syndrome. J Thromb Haemost 2010; 8: 237-242.

29 Pengo V, Ruffatti A, Legnani C, et al. Incidence of a first thromboembolic event in asymptomatic carriers of high risk antiphospholipid antibody profile: a multicenter prospective study. Blood 2011; 118: 4714-4718.

30 Friedman R, Mears JG, Barst RJ. Continuous infusion of prostacyclin normalizes plasma markers of endothelial cell injury and platelet aggregation in primary pulmonary hypertension. Circulation 1997; 96: 2782-2784.

31 Poli D, Grifoni E, Antonucci E, et al. Incidence of recurrent venous thromboembolism and of chronic thromboembolic pulmonary hypertension in patients after a first episode of pulmonary embolism. J Thromb Thrombolysis 2010; 30: 294-299.

32 Auger WR, Fedullo PF. Chronic thromboembolic pulmonary hypertension. Semin Respir Crit Care Med 2009; 30: 471-483.

33 Grifoni S, Vanni S, Magazzini S, et al. Association of persistent right ventricular dysfunction at hospital discharge after acute pulmonary embolism with recurrent thromboembolic events. Arch Intern Med 2006; 166: 2151-2156.

34 Olman MA, Marsh JJ, Lang IM, et al. Endogenous fibrinolytic system in chronic large-vessel thromboembolic pulmonary hypertension. Circulation 1992; 86: 1241-1248.

35 Prandoni P, Prins MH, Lensing AW, et al. Residual thrombosis on ultrasonography to guide the duration of anticoagulation in patients with deep venous thrombosis: a randomized trial. Ann Intern Med 2009; 150: 577-585.

36 Egermayer P, Peacock AJ. Is pulmonary embolism a common cause of chronic pulmonary hypertension? Limitations of the embolic hypothesis. Eur Respir J 2000; 15: 440-448.

37 Bonderman D, Skoro-Sajer N, Jakowitsch J, et al. Predictors of outcome in chronic thromboembolic pulmonary hypertension. Circulation 2007; 115: 2153-2158.

38 Natali D, Jais X, Abraham M, et al. Chronic thromboembolic pulmonary hypertension associated with indwelling Port-A-Cath ${ }_{\mathbb{B}}$ central venous access systems. Am J Respir Crit Care Med 2011; 183: A2409.

39 Langer F, Schramm R, Bauer $\mathrm{M}$, et al. Cytokine response to pulmonary thromboendarterectomy. Chest 2004; 126: 135-141.

40 Kimura H, Okada O, Tanabe N, et al. Plasma monocyte chemoattractant protein-1 and pulmonary vascular resistance in chronic thromboembolic pulmonary hypertension. Am J Respir Crit Care Med 2001; 164: 319-324.

41 Distler JH, Hirth A, Kurowska-Stolarska M, et al. Angiogenic and angiostatic factors in the molecular control of angiogenesis. $Q J$ Nucl Med 2003; 47: 149-161.

42 Waltham M, Burnand KG, Collins M, et al. Vascular endothelial growth factor and basic fibroblast growth factor are found in resolving venous thrombi. J Vasc Surg 2000; 32: 988-996.

43 Firth AL, Yao W, Ogawa A, et al. Multipotent mesenchymal progenitor cells are present in endarterectomized tissues from patients with chronic thromboembolic pulmonary hypertension. Am J Physiol Cell Physiol 2010; 298: C1217-C1225.

44 Sakao S, Hao H, Tanabe N, et al. Endothelial-like cells in chronic thromboembolic pulmonary hypertension: crosstalk with myofibroblast-like cells. Respir Res 2011; 12: 109.

45 Henke PK, Wakefield TW, Kadell AM, et al. Interleukin-8 administration enhances venous thrombosis resolution in a rat model. J Surg Res 2001; 99: 84-91.

46 Waltham M, Burnand KG, Collins M, et al. Vascular endothelial growth factor enhances venous thrombus recanalisation and organisation. Thromb Haemost 2003; 89: 169-176. 
47 Varma MR, Moaveni DM, Dewyer NA, et al. Deep vein thrombosis resolution is not accelerated with increased neovascularization. J Vasc Surg 2004; 40: 536-542.

48 Evans CE, Humphries J, Waltham M, et al. Upregulation of hypoxiainducible factor $1 \alpha$ in local vein wall is associated with enhanced venous thrombus resolution. Thromb Res 2011; 128: 346-351.

49 Morris TA, Marsh JJ, Chiles PG, et al. High prevalence of dysfibrinogenemia among patients with chronic thromboembolic pulmonary hypertension. Blood 2009; 114: 1929-1936.

50 Miniati M, Fiorillo C, Becatti M, et al. Fibrin resistance to lysis in patients with pulmonary hypertension other than thromboembolic. Am J Respir Crit Care Med 2010; 181: 992-996.

51 Morris TA, Marsh JJ, Chiles PG, et al. Fibrin derived from patients with chronic thromboembolic pulmonary hypertension is resistant to lysis. Am J Respir Crit Care Med 2006; 173: 1270-1275.

52 Suntharalingam J, Goldsmith K, van Marion V, et al. Fibrinogen $\mathrm{A} \alpha \mathrm{Thr} 312 \mathrm{Ala}$ polymorphism is associated with chronic thromboembolic pulmonary hypertension. Eur Respir J 2008; 31: 736-741.

53 Le Gal G, Delahousse B, Lacut K, et al. Fibrinogen A $\alpha$-Thr312Ala and factor XIII-A Val34Leu polymorphisms in idiopathic venous thromboembolism. Thromb Res 2007; 121: 333-338.

54 Lang IM, Marsh JJ, Konopka RG, et al. Factors contributing to increased vascular fibrinolytic activity in mongrel dogs. Circulation 1993; 87: 1990-2000.

55 Marsh JJ, Konopka RG, Lang IM, et al. Suppression of thrombolysis in a canine model of pulmonary embolism. Circulation 1994; 90: 3091-3097.

56 Mercier O, Tivane A, Raoux F, et al. A reliable piglet model of chronic thrombo-embolic pulmonary hypertension. Am J Resp Crit Care Med 2011; 183: A2415.

57 Bonderman D, Jakowitsch J, Redwan B, et al. Role for staphylococci in misguided thrombus resolution of chronic thromboembolic pulmonary hypertension. Arterioscler Thromb Vasc Biol 2008; 28: 678-684.
58 Moser KM, Bloor CM. Pulmonary vascular lesions occurring in patients with chronic major vessel thromboembolic pulmonary hypertension. Chest 1993; 103: 685-692.

59 Skoro-Sajer N, Bonderman D, Wiesbauer F, et al. Treprostinil for severe inoperable chronic thromboembolic pulmonary hypertension. J Thromb Haemost 2007; 5: 483-489.

60 Lang IM, Marsh JJ, Olman MA, et al. Expression of type 1 plasminogen activator inhibitor in chronic pulmonary thromboemboli. Circulation 1994; 89: 2715-2721.

61 Lang IM, Marsh JJ, Olman MA, et al. Parallel analysis of tissue-type plasminogen activator and type 1 plasminogen activator inhibitor in plasma and endothelial cells derived from patients with chronic pulmonary thromboemboli. Circulation 1994; 90: 706-712.

62 Galie N, Kim NH. Pulmonary microvascular disease in chronic thromboembolic pulmonary hypertension. Proc Am Thorac Soc 2006; 3: 571-576.

63 Skoro-Sajer N, Hack N, Sadushi-Kolici R, et al. Pulmonary vascular reactivity and prognosis in patients with chronic thromboembolic pulmonary hypertension: a pilot study. Circulation 2009; 119: 298-305.

64 Meneghin A, Hogaboam CM. Infectious disease, the innate immune response, and fibrosis. J Clin Invest 2007; 117: 530-538.

65 Arbustini E, Morbini P, D'Armini AM, et al. Plaque composition in plexogenic and thromboembolic pulmonary hypertension: the critical role of thrombotic material in pultaceous core formation. Heart 2002; 88: 177-182.

66 Maruoka M, Sakao S, Kantake M, et al. Characterization of myofibroblasts in chronic thromboembolic pulmonary hypertension. Int J Cardiol 2011; 159: 119-127.

67 Quarck R, Wynants M, Ronisz A, et al. Characterization of proximal pulmonary arterial cells from chronic thromboembolic pulmonary hypertension patients. Respir Res 2012; 13: 27.

68 Yao W, Firth AL, Sacks RS, et al. Identification of putative endothelial progenitor cells (CD34+CD133+Flk-1+) in endarterectomized tissue of patients with chronic thromboembolic pulmonary hypertension. Am J Physiol Lung Cell Mol Physiol 2009; 296: L870-L878. 